\title{
Diamagnetic Response of Metallic Photonic Crystals at Infrared and Visible Frequencies
}

\author{
Xinhua $\mathrm{Hu},{ }^{1}$ C. T. Chan, ${ }^{2}$ Jian $\mathrm{Zi},{ }^{3}$ Ming Li, ${ }^{1}$ and Kai-Ming $\mathrm{Ho}^{1}$ \\ ${ }^{1}$ Ames Laboratory and Department of Physics and Astronomy, Iowa State University, Ames, Iowa 50011, USA \\ ${ }^{2}$ Department of Physics, Hong Kong University of Science and Technology, Clear Water Bay, Kowloon, Hong Kong, China \\ ${ }^{3}$ Surface Physics Laboratory (National Key Lab), Fudan University, Shanghai 200433, People's Republic of China
}

(Received 28 February 2006; published 5 June 2006)

\begin{abstract}
We show analytically and numerically that diamagnetic response (effective magnetic permeability $\left.\mu_{e}<1\right)$ at infrared and visible frequencies can be achieved in photonic crystals composed of metallic nanowires or nanospheres when the wavelength $\lambda$ is much larger than the lattice constant $a(\lambda \sim 2000 a)$. When $\lambda \sim 100 a$, the metallic photonic crystals will exhibit strong diamagnetic response $\left(\mu_{e}<0.8\right)$, leading to many interesting phenomena such as the unusual Brewster angle for $s$ waves and incidentangle-and-polarization-independent reflection and transmission.
\end{abstract}

DOI: 10.1103/PhysRevLett.96.223901

Photonic crystals (PCs) [1], or periodic composite media, have received great interest due to their unique electromagnetic (EM) properties. A famous example is the photonic band gaps (frequency ranges in which wave propagations are forbidden) which can be utilized to localize and guide light in a unique way [1,2]. Recently, the lowfrequency range much below the first band gap has also attracted growing attention [3-6] due to the occurrence of PCs composed of (carbon) nanotubes [7] or (metallic [8] and semiconductor [9]) nanowires. In the long-wavelength limit (wavelength $\lambda$ much larger than the lattice constant a), these PCs can be viewed as homogenous media described by an effective dielectric constant $\varepsilon_{e}$ and an effective magnetic permeability $\mu_{e}$ [10]. Because of the diversity of microstructures and dielectric constant, $\varepsilon_{e}$ of PCs can be tailored to be of strong anisotropy and of diverse values, resulting in many interesting phenomena that are difficult to be realized in natural crystals, e.g., Dyakonov surface waves [5] and ultraslow guided modes [6].

Conventional materials that exhibit magnetic response $(\mu \neq 1)$ are far less common in nature than materials that exhibit electric response, and they are particularly rare at infrared (ir) and visible frequencies (common ferromagnetic and antiferromagnetic systems work at frequencies below $1 \mathrm{THz}$ ) [11,12]. Consequently, it is very difficult to achieve magnetic response in ir and visible ranges even with composite materials. Recently, Pendry et al. [13] proposed that split rings composed of nonmagnetic metal could exhibit strong magnetic response due to the inherent LC resonance and hence an effectively negative $\mu$ can be obtained at ir and visible frequencies $(\lambda \sim 10 a)$, leading to many novel concepts and potential applications as well [12-15]. However, it is generally believed that usual PCs should not exhibit magnetic response as magnetic resonances do not exist [3-6,10].

In this Letter, we study the long-wavelength behavior of metallic PCs (MPCs) consisting of metallic cylinders or spheres. Analytical formulas are derived for $\varepsilon_{e}$ and $\mu_{e}$ within the coherent-potential approximation (CPA) $[16,17]$ and their accuracy is confirmed by accurate multiple scat-
PACS numbers: 42.70.Qs, 42.25.-p, 78.20.Ci

tering [Korringa-Kohn-Rostoker (KKR)] calculations $[18,19]$. We show that diamagnetic response $\left(\mu_{e}<1\right)$ at ir and visible frequencies can be achieved in the MPCs when the wavelength is still much longer than the unit-cell size $(\lambda \sim 2000 a$ for the $a=150 \mathrm{~nm}$ case $)$. When $\lambda \sim$ $100 a$, MPCs will exhibit strong diamagnetic response $\left(\mu_{e}<0.8\right)$, leading to many interesting phenomena such as the unusual Brewster angle for $s$ waves and incidentangle-and-polarization-independent reflection and transmission. We stress that the magnetic response of our MPCs is different from the metallic split-ring resonators (SRRs) since it does not rely on the magnetic resonances. Although the magnetic response of MPCs is weaker than that of SRRs, it can occur in a wider frequency range.

We first consider a 2D PC composed of circular cylinders of $\left(\varepsilon_{1}, \mu_{1}\right)$ and radius $r$ in the matrix of $(\varepsilon, \mu)$ as shown in Fig. 1(a). For propagation of waves parallel to the plane of periodicity there exist two independent TE and TM modes (the $H$ and $E$ field is parallel to the cylinders, respectively). For TE modes, the magnetic field obeys

$$
\left(\nabla^{2}+k^{2}\right) H_{z}=0,
$$

which is subjected to the continuities of $H_{z}$ and $\frac{1}{\varepsilon} \partial H_{z} / \partial \rho$ at the surface of each cylinder (in the cylindrical coordinates $(\rho, \theta)$ with origin at the center of cylinder). The wave number in the cylinder and the matrix is given by $k_{1}=$ $\sqrt{\varepsilon_{1}} \sqrt{\mu_{1}} k_{0}\left(k_{0}=\omega / c\right.$, where $\omega$ is the angular frequency
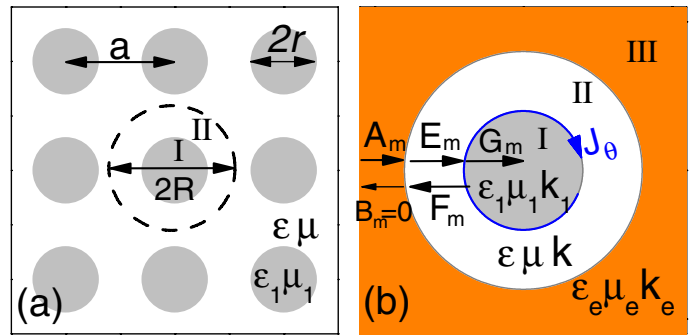

FIG. 1 (color online). (a) A 2D (3D) PC composed of cylinders (spheres) of $\left(\varepsilon_{1}, \mu_{1}\right)$ in the matrix of $(\varepsilon, \mu)$, and (b) a coated cylinder (sphere) in the effective medium of $\left(\varepsilon_{e}, \mu_{e}\right)$. 
and $c$ is the light speed in vacuum) and $k=\sqrt{\varepsilon} \sqrt{\mu} k_{0}$. In the following, we will focus on the TE mode and the results can be applied to TM mode simply by replacing $\left(H_{z}, \varepsilon, \mu\right)$ with $\left(E_{z}, \mu, \varepsilon\right)$.

Then we derive analytical formulas for $\varepsilon_{e}$ and $\mu_{e}$ with the CPA method $[16,17]$ in the long-wavelength limit. We consider a "circular unit cell" (coated cylinder) of radius $R=S_{u} / \sqrt{\pi}$ (where $S_{u}$ is the area of a unit cell; e.g., $S_{u}=$ $a^{2}$ for square lattices. So the filling fraction $f_{s}=\frac{\pi r^{2}}{S_{u}}=\frac{r^{2}}{R^{2}}$ )

$$
-\frac{\frac{1}{\varepsilon} k J_{m}^{\prime}(k R)-J_{m}(k R) \frac{1}{\varepsilon_{e}} k_{e} J_{m}^{\prime}\left(k_{e} R\right) / J_{m}\left(k_{e} R\right)}{\frac{1}{\varepsilon} k H_{m}^{\prime}(k R)-H_{m}(k R) \frac{1}{\varepsilon_{e}} k_{e} J_{m}^{\prime}\left(k_{e} R\right) / J_{m}\left(k_{e} R\right)}
$$

We note that the second term in Eq. (2) is just the $m$ th-order scattering coefficient of the core cylinder [from which the first term can be obtained by replacing $\left(r, k_{1}, \varepsilon_{1}\right)$ by $\left(R, k_{e}, \varepsilon_{e}\right)$ ]. If we only consider the scattering of cylindrical waves of the two lowest orders $m=0$ and $1, \varepsilon_{e}$ and $\mu_{e}$ can be solved numerically from Eq. (2) [by changing Eq. (2) as $\left.C_{m}=\frac{1}{\varepsilon_{e}} k_{e} J_{m}^{\prime}\left(k_{e} R\right) / J_{m}\left(k_{e} R\right)\right]$.

When $k a, k_{e} a \ll 1$, Eq. (2) with $m=0$ and 1 becomes

$$
\begin{gathered}
\mu_{e}=\left(1-f_{s}\right) \mu+f_{s} \tilde{\mu}_{1}, \quad \frac{\varepsilon_{e}-\varepsilon}{\varepsilon_{e}+\varepsilon}=\frac{\tilde{\varepsilon}_{1}-\varepsilon}{\tilde{\varepsilon}_{1}+\varepsilon} f_{s}, \\
\tilde{\mu}_{1}=\mu_{1} p\left(k_{1} r\right), \quad p(x)=-2 J_{0}^{\prime}(x) /\left[x J_{0}(x)\right], \\
\tilde{\varepsilon}_{1}=\varepsilon_{1} g\left(k_{1} r\right), \quad g(x)=J_{1}(x) /\left[x J_{1}^{\prime}(x)\right],
\end{gathered}
$$

where $p(0)=g(0)=1, p(x), g(x) \approx 1$ when $0<|x|<1$, $p(i x) \approx 2 / x$, and $g(i x) \approx 1 / x$ when $x>2$ [20].

For conventional dielectric PCs with comparable $\varepsilon_{1}$ and $\varepsilon, k_{1} r \ll 1$ when $k a \ll 1$ and Eqs. (3)-(5) always reduce to the Maxwell-Garnett (MG) formulas (by $\tilde{\mu}_{1}=\mu_{1}$ and $\tilde{\varepsilon}_{1}=\varepsilon_{1}$ ) [10], namely

$$
\mu_{e}=\left(1-f_{s}\right) \mu+f_{s} \mu_{1}, \quad \frac{\varepsilon_{e}-\varepsilon}{\varepsilon_{e}+\varepsilon}=\frac{\varepsilon_{1}-\varepsilon}{\varepsilon_{1}+\varepsilon} f_{s} .
$$

But in MPCs, $k_{1} r \ll 1$ only when $k a \rightarrow 0 \quad\left[\varepsilon_{1}=\right.$ $1-f_{p}^{2} /\left(f^{2}+i f_{\tau} f\right) \approx i f_{p}^{2} / f_{\tau} f$ when $\left.f \rightarrow 0\right]$. When $k a$ is not so small $\left(\varepsilon_{1} \approx-f_{p}^{2} / f^{2}\right), k_{1} r \approx i r / \delta_{p}$, where $\delta_{p}=$ $c / 2 \pi f_{p}$ is the skin depth of metal ( $\sim 13 \mathrm{~nm}$ for $\left.\mathrm{Al}\right)$ in the ir or visible range. $\tilde{\mu}_{1} \approx \mu_{1}$ and the MG formulas will be valid only when $r<\delta_{p}$. When $r>\delta_{p}, \tilde{\mu}_{1}<\mu_{1}$ and MPCs will be effectively diamagnetic. Especially when $r>40 \delta_{p}$ and $\lambda<100 a$ (to be shown later), $\tilde{\mu}_{1} \approx 0$ and the MPCs will exhibit strong diamagnetic response.

There exists a particular case of perfect metallic PCs (PMPCs) [3]. Using $\mu_{1}=1$ and $\varepsilon_{1}=-f_{p}^{2} / f^{2}\left(f_{p} \rightarrow+\infty\right)$, Equations (3)-(5) become (by $\tilde{\mu}_{1}=0$ and $\tilde{\varepsilon}_{1}=-\infty$ ):

$$
\mu_{e}=\left(1-f_{s}\right) \mu, \quad \varepsilon_{e}=\varepsilon\left(1+f_{s}\right) /\left(1-f_{s}\right) .
$$

Equation (7) can also be derived using the CPA method and the boundary condition of $\partial H_{z}^{I I}(r) / \partial \rho=0$ (TE) for perfect-metal cylinders [17]. Previously, Nicorovici et al. [3] found an interesting problem in PMPCs of $\varepsilon=\mu=1$ that the refraction index $n_{e}=\sqrt{1+f_{s}}$ could not be ex- and replace the 2D PC outside by a uniform effective medium of $\varepsilon_{e}, \mu_{e}$, and $k_{e}=\sqrt{\varepsilon_{e}} \sqrt{\mu_{e}} k_{0}$ [see Fig. 1(b)]. $H_{z}$ can be written as: $H_{z}^{I}=\sum_{m} G_{m} J_{m}\left(k_{1} \rho\right) e^{i m \theta}$ when $\rho<$ $r, H_{z}^{I I}=\sum_{m}\left[E_{m} J_{m}(k \rho)+F_{m} H_{m}(k \rho)\right] e^{i m \theta}$ when $R>\rho>$ $r$, and $H_{z}^{I I I}=\sum_{m}\left[A_{m} J_{m}\left(k_{e} \rho\right)+B_{m} H_{m}\left(k_{e} \rho\right)\right] e^{i m \theta}$ when $\rho>R$ [where the Bessel (Hankel) function $J_{m}\left(H_{m}\right)$ stands for the $m$ th-order cylindrical incident (scattering) waves]. It can be shown that the $m$ th-order scattering coefficient of the coated cylinder $D_{m} \equiv B_{m} / A_{m}=0$ (which defines the effective medium) when

$=-\frac{\frac{1}{\varepsilon} k J_{m}^{\prime}(k r)-J_{m}(k r) \frac{1}{\varepsilon_{1}} k_{1} J_{m}^{\prime}\left(k_{1} r\right) / J_{m}\left(k_{1} r\right)}{\frac{1}{\varepsilon} k H_{m}^{\prime}(k r)-H_{m}(k r) \frac{1}{\varepsilon_{1}} k_{1} J_{m}^{\prime}\left(k_{1} r\right) / J_{m}\left(k_{1} r\right)}$

plained using the MG theory and $\varepsilon_{1}=-\infty$. Now the problem is clear that $n_{e}=\sqrt{\varepsilon_{e}^{-}} \sqrt{\mu_{e}}$ and Eq. (7) should be used for PMPCs, agreeing with the recent suggestion by Krokhin and Reyes [3].

To check the validity of the above analytic formulas, we use the $S$-matrix-combined KKR method (including highorder cylindrical waves) $[18,19]$ and do the transmission calculations for the normal incidence of a plane wave upon a MPC slab. Then the $\varepsilon_{e}$ and $\mu_{e}$ of MPCs can be obtained from the complex transmission and reflection coefficients [14,21]. In Fig. 2, we show the accurate KKR results of $\varepsilon_{e}$ and $\mu_{e}$ for a square lattice of Al cylinders in air with $a=$ $150 \mathrm{~nm}$ and $r=52.5 \mathrm{~nm}$ together with those from different formulas. It can be seen that the MG formulas are only valid when $\lambda>2000 a$ and the MPCs are nonmagnetic. For higher frequencies, the MPCs become effectively diamagnetic and Eqs. (3) $-(5)$ (when $120 a<\lambda<2000 a$ ) or direct solution of Eq. (2) (when $5 a<\lambda<120 a$ ) should be used.
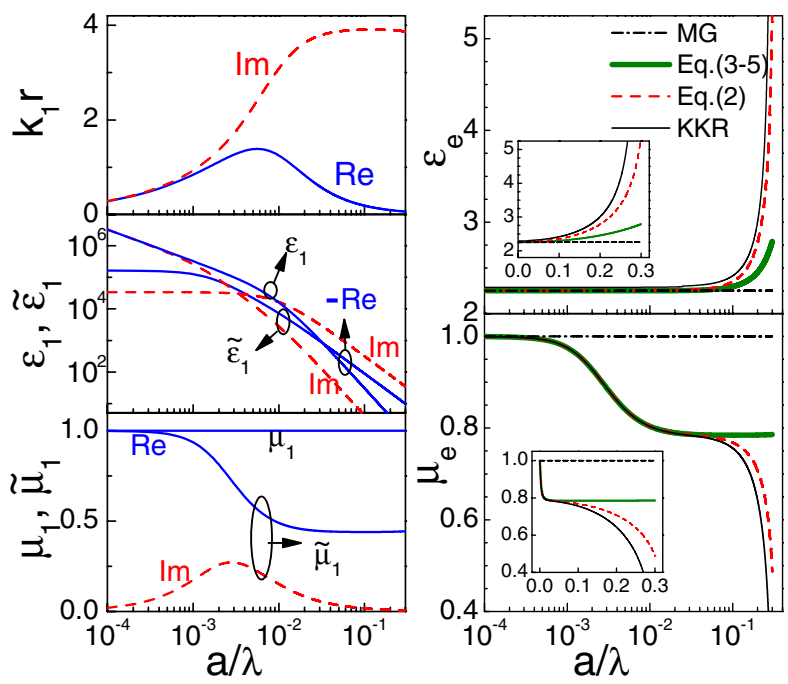

FIG. 2 (color online). $k_{1} r, \varepsilon_{1}, \tilde{\varepsilon}_{1}, \mu_{1}, \tilde{\mu}_{1}, \varepsilon_{e}$ (real part), and $\mu_{e}$ (real part) of a 2D PC (square lattice) of aluminum cylinders in air in the lowest TE band $\left[0<\operatorname{Im}\left(\varepsilon_{e}\right)<0.04,0<\operatorname{Im}\left(\mu_{e}\right)<\right.$ 0.09]. The lattice constant $a=150 \mathrm{~nm}$ and the radius of cylinder $r=52.5 \mathrm{~nm} . \mu_{1}=1$ and a Drude model of $\varepsilon_{1}=$ $1-f_{p}^{2} /\left(f^{2}+i f_{\tau} f\right)$ are used for Al where $f_{p}=3570 \mathrm{THz}$, $f_{\tau}=19.4 \mathrm{THz}$. 

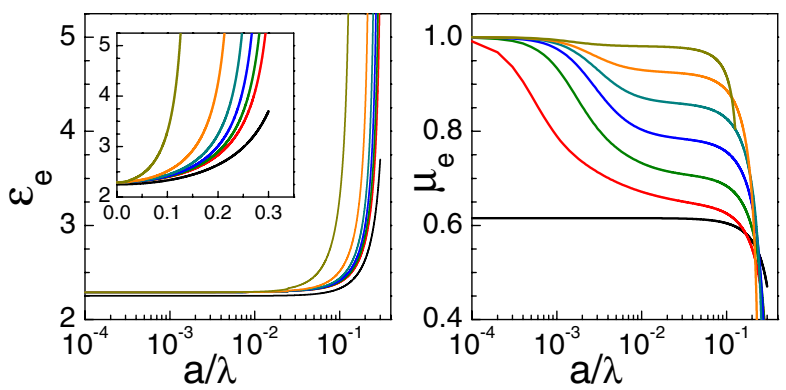

FIG. 3 (color online). KKR results of $\varepsilon_{e}$ and $\mu_{e}$ of 2D PCs (square lattice) of $\mathrm{Al}$ (from top to bottom: the lattice constant $a=25,55,90,150,300,1000 \mathrm{~nm}$ ) or perfect-metal (the bottom line) cylinders $(r / a=0.35)$ in air for TE mode.

The MPC will be strongly effectively diamagnetic $\left(\mu_{e}<\right.$ 0.8 ) when $\lambda<100 a$. When $\lambda<120 a$ (approaching the band gap), $\mu_{e}$ will decrease strongly and diverge at the band edge due to the Bragg resonance. At high frequencies near the band gap $(\lambda<5 a)$, the scattering of high-order cylindrical waves will be important and more accurate KKR calculations are needed.

In Fig. 3, we show the KKR results of $\varepsilon_{e}$ and $\mu_{e}$ for square lattices of $\mathrm{Al}$ and perfect-metal cylinders $(r / a=$ 0.35 ) in air. It can be seen that the MPCs have almost the same $\varepsilon_{e} \approx 2.28$ when $\lambda>50 a$. But $\mu_{e}$ will be quite different for MPCs with varying the lattice constant $a$. When $\lambda>10 a$, the MPC with $a<25 \mathrm{~nm}$ (so $r<0.7 \delta_{p}$ ) behaves nonmagneticly and those with larger $a$ can be effectively diamagnetic. When $a>1.5 \mu \mathrm{m}$ (so $r>40 \delta_{p}$ ), the MPC can have almost the same diamagnetic property with PMPC for $\lambda<100 a$.

The $\varepsilon_{e}$ and $\mu_{e}$ of MPCs can be used to determine the optical properties of MPCs, such as refraction, reflection, and transmission [11]. Here we will focus on the Brewster angle $\left(\theta_{b}\right)$ phenomenon, i.e., total transmission of $\mathrm{TE}$ waves at a particular incident angle $\theta_{b}$. In usual dielectric materials, the zero reflection occurs when the reflected rays are perpendicular to the refracted rays due to the zero EM emission of electric dipoles (excited by refracted waves) in the dipolar direction. But for the MPCs with $\mu_{e}<1$, magnetic dipoles will also be excited and this perpendicularity does not exist. It can be shown that

$$
\theta_{b}=\arctan \sqrt{\left(\mu_{b} \varepsilon_{e}^{2}-\varepsilon_{b} \varepsilon_{e} \mu_{e}\right) /\left(\varepsilon_{b} \varepsilon_{e} \mu_{e}-\mu_{b} \varepsilon_{b}^{2}\right)}
$$

for a MPC in the background with $\varepsilon_{b}$ and $\mu_{b}$ [11]. In Fig. 4, we show the Brewster angle for a square lattice of
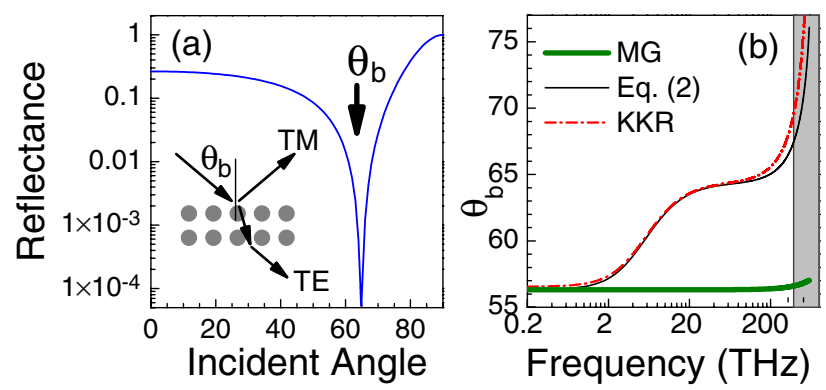

FIG. 4 (color online). (a) Reflectance as a function of the incident angle $\theta$ at $\lambda=1550 \mathrm{~nm}$ (TE incidence), and (b) Brewster angle as a function of the frequency of incident waves for a two layer of the 2D MPC studied in Fig. 2 (the transmittance is less than $5 \times 10^{-9}$ for the TM incidence). The light shaded range stands for the visible frequencies.

Al cylinders in air $(a=150 \mathrm{~nm}, r=52.5 \mathrm{~nm})$. The $\theta_{b}$ predicted by Eqs. (2) and (8) agrees well with the accurate KKR value. For frequencies lower than $1 \mathrm{THz}$, the MPC will be nonmagnetic $\left(\varepsilon_{e}=2.28, \mu_{e} \approx 1\right)$ and $\theta_{b} \approx 56.5^{\circ}$. When $f=193 \mathrm{THz}(\lambda=1550 \mathrm{~nm})$, the MPC will exhibit strong diamagnetic response and $\theta_{b}=65.5^{\circ}$. Previously, 2D MPCs can act as ir polarizers (usually working at normal incidence) due to the low-frequency band gap for TM waves. Our results indicate that 2D MPCs can further act as ir TE-TM splitters at $\theta_{b}$. We note that this complete splitting of TE and TM waves does not exist at dielectric interfaces.

By now, we have shown the effectively diamagnetic behavior of 2D MPCs for the TE waves. This diamagnetic response can be understood by $\mu_{e} \equiv\left\langle B_{z}\right\rangle / \mu_{0}\left\langle H_{z}\right\rangle=(1-$ $\left.f_{s}\right) \mu+f_{s} \mu_{1}\left\langle H_{z}^{I}\right\rangle / H_{z}^{I I}$, where $\left\langle H_{z}\right\rangle=H_{z}^{I I} \quad\left(\left\langle B_{z}\right\rangle=\right.$ $\left.\left(1-f_{s}\right) B_{z}^{I I}+f_{s}\left\langle B_{z}^{I}\right\rangle\right)$ is the average of $H(B)$ field over the line-boundary (surface) of the unit cell [14]. For $m=$ $0, H_{z}^{I}=J_{0}\left(k_{1} \rho\right), H_{z}^{I I}=H_{z}^{I}(r)=J_{0}\left(k_{1} r\right)($ when $k a \ll 1)$, $\left\langle H_{z}^{I}\right\rangle \equiv 2 r^{-2} \int_{0}^{r} H_{z}^{I} \rho d \rho=-2 J_{0}^{\prime}\left(k_{1} r\right) / k_{1} r$, and thus the $\mu_{e}$ in Eq. (3) can be alternatively obtained. We note that surface currents $J_{\theta}=\left(\varepsilon_{1}^{-1}-1\right)\left[\nabla \times \mathbf{H}^{I}\right]_{\theta} \approx-\partial H_{z}^{I} / \partial \rho$ can be induced in metal cylinders [see Fig. 1(b)] and $\left\langle H_{z}^{I}\right\rangle=-2 J_{0}^{\prime}\left(k_{1} r\right) / k_{1} r$ can also be obtained by $\left\langle H_{z}^{I}\right\rangle=$ (external) $H_{z}^{I I}+$ (induced) $r^{-2} \int_{0}^{r} J_{\theta} \rho^{2} d \rho$. Since $H_{z}^{I}$ decays inside the metallic cylinders and $\left\langle H_{z}^{I}\right\rangle<H_{z}^{I I}, \mu_{e}$ can be less than 1 in MPCs.

Similar effective diamagnetic response can also exist in 3D MPCs consisting of metallic spheres. Unlike 2D, 3D MPCs can have isotropic $\mu_{e}$ and $\varepsilon_{e}$ due to degenerated TE and TM modes at the low frequencies. Using similar CPA derivations, we can obtain the following relation

$$
\frac{\frac{1}{\mu} \frac{\partial}{\partial R}\left[R j_{l}(k R)\right]-j_{l}(k R) \frac{1}{\mu_{e}} \frac{\partial}{\partial R}\left[R j_{l}\left(k_{e} R\right)\right] / j_{l}\left(k_{e} R\right)}{\frac{1}{\mu} \frac{\partial}{\partial R}\left[R h_{l}(k R)\right]-h_{l}(k R) \frac{1}{\mu_{e}} \frac{\partial}{\partial R}\left[R j_{l}\left(k_{e} R\right)\right] / j_{l}\left(k_{e} R\right)}
$$

$=\frac{\frac{1}{\mu} \frac{\partial}{\partial r}\left[r j_{l}(k r)\right]-j_{l}(k r) \frac{1}{\mu_{1}} \frac{\partial}{\partial r}\left[r j_{l}\left(k_{1} r\right)\right] / j_{l}\left(k_{1} r\right)}{\frac{1}{\mu} \frac{\partial}{\partial r}\left[r h_{l}(k r)\right]-h_{l}(k r) \frac{1}{\mu_{1}} \frac{\partial}{\partial r}\left[r j_{l}\left(k_{1} r\right)\right] / j_{l}\left(k_{1} r\right)}$

and another equation with replacing $\mu$ by $\varepsilon$ from the zero $H$ and $E$ scattering [22], where $j_{l}\left(h_{l}\right)$ is the $l$ th spherical Bessel (Hankel) function. When $k a, k_{e} a \ll 1$, the equations with $l=1$ (the lowest order in 3D) become

$$
\begin{gathered}
\frac{\mu_{e}-\mu}{\mu_{e}+2 \mu}=f_{s} \frac{\tilde{\mu}_{1}-\mu}{\tilde{\mu}_{1}+2 \mu}, \quad \frac{\varepsilon_{e}-\varepsilon}{\varepsilon_{e}+2 \varepsilon}=f_{s} \frac{\tilde{\varepsilon}_{1}-\varepsilon}{\tilde{\varepsilon}_{1}+2 \varepsilon} \\
\tilde{\mu}_{1}=\mu_{1} q\left(k_{1} r\right), \quad \tilde{\varepsilon}_{1}=\varepsilon_{1} q\left(k_{1} r\right)
\end{gathered}
$$



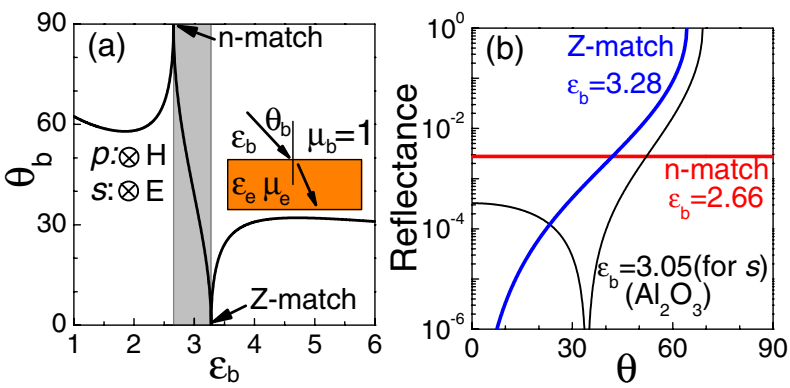

FIG. 5 (color online). (a) Brewster angle as a function of $\varepsilon_{b}$ (the light gray area is for $s$ waves and others for $p$ waves), and (b) reflectance as a function of incident angle $\theta$ for the incidence of light at $\lambda=1550 \mathrm{~nm}$ from a dielectric media of $\varepsilon_{b}$ to a $3 \mathrm{D}$ MPC of $\varepsilon_{e}=2.95$ and $\mu_{e}=0.90$.

$$
q(x)=2 /\left[1+x j_{1}^{\prime}(x) / j_{1}(x)\right],
$$

where $f_{s}$ is the volume ratio of spheres in PCs $[15,20]$. Since $q(x)$ behaves like $p(x)$, similar diamagnetic response (like Fig. 3) can occur in 3D MPCs in the long-wavelength limit.

It is known that the Brewster angle exists only for $p$ waves at usual dielectric interfaces. However, at the interface between a dielectric medium of $\varepsilon_{b}$ and a 3D MPC of $\varepsilon_{e}$ and $\mu_{e}\left(\mu_{e}<1\right)$, the Brewster angle can also exist for $s$ waves when $\varepsilon_{e} \mu_{e}<\varepsilon_{b}<\varepsilon_{e} / \mu_{e}$ (for $p$ waves when $\varepsilon_{b}<$ $\varepsilon_{e} \mu_{e}$ or $\left.\varepsilon_{b}>\varepsilon_{e} / \mu_{e}\right)$ [11]. For example, a simple-cubic lattice $(a=150 \mathrm{~nm})$ of Al spheres of $r=48.5 \mathrm{~nm}$ in glass $(\varepsilon=2)$ will be of $\mu_{e}=0.90$ and $\varepsilon_{e}=2.95$ at $\lambda=$ $1550 \mathrm{~nm}$. When $2.66<\varepsilon_{b}<3.28$, a Brewster angle exists for $s$ waves (see Fig. 5); e.g., $\theta_{b}=34.4^{\circ}$ for $\varepsilon_{b}=3.05$ $\left(\mathrm{Al}_{2} \mathrm{O}_{3}\right)$. When $\varepsilon_{b}=\varepsilon_{e} / \mu_{e}=3.28$ (impedance match), $\theta_{b}=0^{\circ}$ and both $s$ and $p$ waves can totally enter the 3D MPC at normal incidence.

The reflection and transmission depend on both the incident angle and polarizations at dielectric interfaces. But at the interface between a dielectric medium of $\varepsilon_{b}$ and a 3D MPC of $\varepsilon_{e}$ and $\mu_{e}$, the dependence will not exist when the refractive indices are matched, namely $\varepsilon_{b}=\varepsilon_{e} \mu_{e}$ [see Fig. 5(b), where the reflectance can be increased using a 3D MPC of larger $f_{s}$ ]. This interesting feature may facilitate the fabrication of some incidentangle-and-polarization-independent optical devices [23].

In summary, we have demonstrated effective diamagnetic response of MPCs in the very long-wavelength range $(\lambda<2000 a)$ by nontrivial modifications of the MaxwellGarnett formulas, leading to many interesting phenomena such as the unusual Brewster angle for $s$ waves and incident-angle-and-polarization-independent reflection and transmission.

X. H. thanks Dr. N.A. Nicorovici for discussions on the lattice sums in Refs. [18,19] and providing an erratum of Ref. [18]. We also thank Dr. Zhao-Qing Zhang, Dr. Hongqiang Li, and Dr. Jensen Li for helpful discussions. This work is supported by the Director for Energy Research, Office of Basic Energy Sciences. J. Z. acknowl- edges support from CNKBRSF and NSFC. The Ames Laboratory is operated for the U. S. Department of Energy by Iowa State University under Contract No. W-7405ENG-82.

[1] E. Yablonovitch, Phys. Rev. Lett. 58, 2059 (1987); S. John, Phys. Rev. Lett. 58, 2486 (1987).

[2] K. Sakoda, Optical Properties of Photonic Crystals (Springer, Berlin, 2001).

[3] N.A. Nicorovici, R. C. McPhedran, and L.C. Botten, Phys. Rev. Lett. 75, 1507 (1995); A. A. Krokhin and E. Reyes, Phys. Rev. Lett. 93, 023904 (2004).

[4] F. J. Garcia-Vidal, J. M. Pitarke, and J. B. Pendry, Phys. Rev. Lett. 78, 4289 (1997).

[5] P. Halevi, A. A. Krokhin, and J. Arriaga, Phys. Rev. Lett. 82, 719 (1999); D. Artigas and L. Torner, Phys. Rev. Lett. 94, 013901 (2005).

[6] J. T. Shen, P. B. Catrysse, and S. Fan, Phys. Rev. Lett. 94, 197401 (2005).

[7] S. Iijima, Nature (London) 354, 56 (1991); K. Jiang, Q. Li, and S. Fan, Nature (London) 419, 801 (2002).

[8] B. H. Hong et al., Science 294, 348 (2001); G. Sauer et al., J. Appl. Phys. 91, 3243 (2002); Y. T. Pang et al., Nanotechnology 14, 20 (2003); X. Hu and C. T. Chan, Appl. Phys. Lett. 85, 1520 (2004); X. Ao and S. He, Appl. Phys. Lett. 87, 101112 (2005).

[9] M.H. Huang et al., Science 292, 1897 (2001).

[10] J. C. Maxwell Garnett, Philos. Trans. R. Soc. London 203, 385 (1904); Electrical Transport and Optical Properties of Inhomogenous Media, edited by J.C. Garland and D. B. Tanner (AIP, New York, 1978); D. E. Aspnes, Am. J. Phys. 50, 704 (1982).

[11] L. D. Landau and E. M. Lifshitz, Electrodynamics of Continuous Media (Pergamon Press, Oxford, 1984).

[12] T. J. Yen et al., Science 303, 1494 (2004).

[13] J. B. Pendry et al., IEEE Trans. Microwave Theory Tech. 47, 2075 (1999); R. A. Shelby et al., Science 292, 77 (2001); S. Linden et al., Science 306, 1351 (2004); J. Zhou et al., Phys. Rev. Lett. 95, 223902 (2005).

[14] S. O'Brien and J. B. Pendry, J. Phys. Condens. Matter 14, 4035 (2002).

[15] A. K. Sarychev, R.C. McPhedran, and V.M. Shalaev, Phys. Rev. B 62, 8531 (2000).

[16] P. Sheng, Introduction to Wave Scattering, Localization, and Mesoscopic Phenomena (Academic Press, New York, 1995).

[17] X. Hu and C. T. Chan, Phys. Rev. Lett. 95, 154501 (2005).

[18] L. C. Botten et al., Phys. Rev. E 64, 046603 (2001); L. C. Botten et al., J. Opt. Soc. Am. A 17, 2165 (2000).

[19] V. Twersky, Arch. Ration. Mech. Anal. 8, 323 (1961).

[20] When $x \rightarrow 0, J_{0}(x)=1, J_{0}^{\prime}(x)=-J_{1}(x)=-\frac{x}{2}, J_{1}^{\prime}(x)=$ $\frac{1}{2}, H_{0}(x)=\frac{2 i}{\pi} \ln \left(\frac{x}{2}\right), H_{0}^{\prime}(x)=-H_{1}(x)=\frac{2 i}{\pi x}, H_{1}^{\prime}(x)=\frac{2 i}{\pi x^{2}}$, $j_{1}(x)=\frac{x}{3}, j_{1}^{\prime}(x)=\frac{1}{3}, h_{1}(x)=-\frac{i}{x^{2}}, h_{1}^{\prime}(x)=\frac{2 i}{x^{3}}$.

[21] X. Hu, C. T. Chan, and J. Zi, Phys. Rev. E 71, 055601(R) (2005).

[22] N. Stefanou, V. Yannopapas, and A. Modinos, Comput. Phys. Commun. 113, 49 (1998).

[23] E. Lidorikis et al., Phys. Rev. Lett. 91, 023902 (2003). 